(C) 1997 Vilniaus Gedimino technikos universitetas

\title{
ON GENERAL REPRESENTATION OF THE MEROMORPHIC SOLUTIONS OF HIGHER ANALOGUES OF THE SECOND PAINLEVE EQUATION
}

\author{
V. I. GROMAK and T. S. STEPANOVA
}

Belarussian State University

Fr.Skorina Avenue 4, 220050 Minsk, Belarus

E:mail:grom@mmf.bsu.unibel.by

One of the important questions of the nonlinear ordinary differential equations theory is representation of the meromorphic solutions as the ratio of the entire functions similarly to the Weierstrass function $\rho(z)$, which is a solution of an equation ${\rho^{\prime}}^{2}=4 \rho^{3}+g_{2} \rho+g_{3}$, and it has representation through the entire function $\sigma(z)$

$$
\rho(z)=\frac{\sigma^{\prime^{2}}-\sigma \sigma^{\prime \prime}}{\sigma^{2}}=-\zeta^{\prime}(z), \quad \zeta=\left(\ln (\sigma(z))^{\prime}\right.
$$

We shall consider a reduction of the higher - Korteweg-de Vries equations

$$
(2 m-1) u_{t}=X_{m} u
$$

where $X_{1} u=D u=D \frac{\partial H_{1}}{\partial u}, \quad D=\frac{\partial}{\partial x}, \quad X_{m} u=\left(2 u+2 D u D^{-1}-D^{2}\right) X_{m-1} u=$ $D \frac{\partial H_{m}}{\partial u}, \quad m=2,3, \ldots$

applying the formulas

$$
z=x t^{-\frac{1}{2 m-1}}, \quad u(x, t)=t^{-\frac{2}{2 m-1}}\left(\frac{d w}{d z}+w^{2}\right)
$$

to the ordinary differential equation

$$
D^{-1} S_{w}^{m-1}\left(w^{\prime}\right)+z w+\delta=0, \quad\left({ }_{m} P_{2}\right)
$$


where $S_{w}=4 w^{2}+4 w^{\prime} D_{w}^{-1}-D^{2}, \quad D=d / d z$.

The order of equation $\left({ }_{m} P_{2}\right)$ is $2 m-2$. For $m=2$ equations $(1)$ and $\left({ }_{m} P_{2}\right)$ become the Korteweg - de Vries equation and the second Painleve equation $\left(P_{2}\right)$ correspondingly. Let us call equation $\left({ }_{m} P_{2}\right)$ the higher analogue of the second Painleve equation similarly to equation (1). For $m=3$ we have

$$
w^{(4)}=10 w^{2} w^{\prime \prime}+10 w w^{\prime^{2}}-6 w^{5}-z w-\delta .
$$

It is well known, that solutions of the Painleve equations are, in a general case, meromorphic functions, which we may not state definitely about the higher analogues of equation $P_{2}$. In paper [1] representation of the Painleve equations' solutions as the ratio of the entire functions was given and one-toone correspondence between the solutions of these equations and the systems constructed for them was established.

In the present paper we offer representation of the meromorphic solutions of equations $\left({ }_{m} P_{2}\right)$ as the ratio

$$
w(z)=\frac{v(z)}{u(z)}
$$

of entire functions $v(z), u(z)$. We establish one-to-one correspondence between the meromorphic solutions of the equation $\left({ }_{m} P_{2}\right)$ and entire solutions of the constructed system.

For the equation $\left({ }_{m} P_{2}\right)$ let's assume following $[2,3,1]$

$$
u(z)=\exp \left(-\int_{z_{0}}^{z} d z \int_{z_{0}}^{z} w^{2} d z\right)
$$

where the path of integration does not pass through the singularities of the function $w(z)$. In the neighborhood of the movable pole $z=\alpha$ the meromorphic solution $w(z)$ has expansion [4]

$$
w=a_{-1}(z-\alpha)^{-1}+a_{1}(z-\alpha)+\varphi_{1}(z, \alpha),
$$

where $a_{-1}$ takes any of the values $\pm 1, \pm 2, \ldots \pm(m-1)$, and $\varphi_{1}(z, \alpha)$ is an analytic function in the neighborhood of $\alpha$. Then from (4) it follows that $u(z)$ is an entire function for any meromorphic solution of the equation $\left({ }_{m} P_{2}\right)$, and at pole $\alpha$ of the solution $w(z)$ the function $u(z)$ has zero of order $a_{-1}^{2}$. This fact is easily established by substitution (5) into the right part of (4). Hence, if we will define function $v(z)$ as $v(z)=w(z) u(z)$, then the function $v(z)$ is also an entire one for any meromorphic solution of the equation $\left({ }_{m} P_{2}\right)$. The system for finding of the entire functions $u(z), v(z)$ turns out by differentiation (4) by virtue of (3) and substitution (3) into the equation $\left({ }_{m} P_{2}\right)$. It has the form

$$
u u^{\prime \prime}-u^{\prime^{2}}=v^{2}, \quad D^{-1} S_{v u^{-1}}^{m-1}\left(v^{\prime} u^{-1}-v u^{\prime} u^{-2}\right)+z v u^{-1}+\delta=0 .
$$


For example, at $m=3$ for the equation $\left({ }_{3} P_{2}\right)$ we have

$$
\begin{aligned}
& u u^{\prime \prime}-u^{\prime^{2}}=v^{2} \\
& v^{(4)} u^{4}-4 v^{\prime \prime} v^{\prime} u^{3}+6 v^{\prime \prime} u^{\prime^{2}} u^{2}-2 v^{\prime \prime} v^{2} u^{2}-4 v^{\prime} u^{3^{3}} u \\
& \quad+4 v^{2} v^{\prime} u^{\prime}+v u^{\prime^{4}}-2 v^{3} u^{\prime^{2}}+v^{5}+z v u^{4}+\delta u^{5}=0 .
\end{aligned}
$$

Let us consider system (6).

Lemma 1. The system (6) has the solution

$$
v=0, \quad u=\exp (a z+b)
$$

for any $a, b \in C, \delta=0$.

The choice of the solution of system (6) is defined by the initial conditions $u\left(z_{0}\right)=u_{0}, u^{\prime}\left(z_{0}\right)=u_{0}^{\prime}, v\left(z_{0}\right)=v_{0}, v^{\prime}\left(z_{0}\right)=v_{0}^{\prime}, \ldots, v^{(2 m-3)}\left(z_{0}\right)=v_{0}^{(2 m-3)}$,

where $z_{0}, \quad u_{0}, \quad u_{0}^{\prime}, \quad v_{0}, v_{0}^{\prime}, \ldots, \quad v_{0}^{(2 m-3)} \in C$. Thus, if $u\left(z_{0}\right)=0$, then the initial conditions (8) is singular.

Lemma 2. If $(v, u)$ is a solution of system (6), then

$$
(\tilde{v}, \tilde{u})=(\lambda(z) v, \lambda(z) u), \quad \lambda(z) \not \equiv 0
$$

is a solution of system (6) if and only if $\lambda=e^{a z+b}, \quad a, b \in C$.

The validity of lemmas 1 and 2 are confirmed by direct substitution (7) and (9) into system (6).

Lemma 3. Any solution of system (6), corresponding to the meromorphic solution of an equation $\left({ }_{m} P_{2}\right)$ and satisfying to the initial conditions

$u\left(z_{0}\right)=1, \quad u^{\prime}\left(z_{0}\right)=0, \quad v\left(z_{0}\right)=v_{0}, \quad v^{\prime}\left(z_{0}\right)=v_{0}^{\prime}, \ldots, \quad v^{(2 m-3)}\left(z_{0}\right)=v_{0}^{(2 m-3)}$,

is an entire one.

$\mathrm{P} \mathrm{r}$ o o f. The initial conditions (10) are not singular. Let $w(z)$ be a meromorphic solution of an equation $\left({ }_{m} P_{2}\right)$ with the initial conditions $w\left(z_{0}\right)=$ $w_{0}, w^{\prime}\left(z_{0}\right)=w_{0}^{\prime}, \ldots, w^{(2 m-3)}\left(z_{0}\right)=w_{0}^{(2 m-3)}$. We shall consider the functions

$$
u_{1}(z)=\exp \left(-\int_{z_{0}}^{z} d z \int_{z_{0}}^{z} w^{2} d z\right), \quad v_{1}(z)=w(z) u_{1}(z) .
$$

From construction functions $u_{1}(z), v_{1}(z)$ are the entire ones and satisfy to system (6). By virtue of uniqueness the statement will be proved if we take $w_{0}=v_{0}, w_{0}^{\prime}=v_{0}^{\prime}, \ldots, w_{0}^{(2 m-3)}=v_{0}^{(2 m-3)}$. 
Lemma 4. Any solution of system (6), corresponding to the meromorphic solution of equation $\left({ }_{m} P_{2}\right)$ and satisfying to the initial conditions

$$
\begin{aligned}
& u\left(z_{0}\right)=u_{0} \neq 0, \quad u^{\prime}\left(z_{0}\right)=u_{0}^{\prime}, \quad v\left(z_{0}\right)=v_{0}, \quad v^{\prime}\left(z_{0}\right)=v_{0}^{\prime}, \ldots, \\
& v^{(2 m-3)}\left(z_{0}\right)=v_{0}^{(2 m-3)},
\end{aligned}
$$

is an entire one.

P r o o f. We shall take the solution $(\tilde{v}, \tilde{u})$ of system (6) with the initial conditions

$\tilde{u}\left(z_{0}\right)=1, \quad \tilde{u}^{\prime}\left(z_{0}\right)=0, \quad \tilde{v}\left(z_{0}\right)=\tilde{v}_{0}, \quad \tilde{v}^{\prime}\left(z_{0}\right)=\tilde{v}_{0}^{\prime}, \ldots, \quad \tilde{v}^{(2 m-3)}\left(z_{0}\right)=\tilde{v}_{0}^{(2 m-3)}$.

By virtue of lemma3 this solution is an entire one, and by virtue of lemma 2 the functions $(v, u)=(\tilde{v} \exp (a z+b), \tilde{u} \exp (a z+b))$ will also be an entire solution of system (6). The proof of lemma 4 follows from the choice $a, b$ and $\tilde{v}_{0}, \tilde{v}_{0}^{\prime}, \ldots, \tilde{v}_{0}^{(2 m-3)}$ from a condition

$$
a=\frac{u_{0}^{\prime}}{u_{0}}, b=\ln \left(u_{0}\right)-z_{0} \frac{u_{0}^{\prime}}{u_{0}}, \tilde{v}_{0}=\frac{v_{0}}{u_{0}}, \ldots, \quad \tilde{v}_{0}^{(2 m-3)}=\left(\frac{v}{u}\right)^{(2 m-3)}\left(z_{0}\right),
$$

where $u^{\prime \prime}\left(z_{0}\right)=u_{0}^{\prime 2} / u_{0}$.

THEOREM 1. All solutions of system (6), corresponding to the meromorphic solutions of equation $\left({ }_{m} P_{2}\right)$, are entire functions.

$\mathrm{P}$ r o o f. If $u \equiv 0$, then $v \equiv 0$ and this solution is an entire one. Let $u \not \equiv 0$. Then there exists domain $D$ where function $u(z) \neq 0$ and it is an analytic one. Let $z_{0} \in D$. Then by virtue of lemma 4 we have the required statement.

THEOREM 2. Let $(v, u)$ be an arbitrary entire non-zero solution of system (6) for some fixed value of parameter $\delta$, which is different from the solutions (7). Then a ratio $v(z) / u(z)$ represents meromorphic solution of equation $\left({ }_{m} P_{2}\right)$ for the same value of parameter $\delta$.

$\mathrm{P}$ r o o f. Let $(v, u)$ be an entire non-zero solution of system (6). Then there exists such $z_{0}$, that $u\left(z_{0}\right)=u_{0} \neq 0$. Let us take the meromorphic solution $w(z)$ of equation $\left({ }_{m} P_{2}\right)$ with the initial conditions $w\left(z_{0}\right)=v_{0} / u_{0}, \quad w^{\prime}\left(z_{0}\right)=$ $v_{0}^{\prime} / u_{0}-v_{0} u_{0}^{\prime} / u_{0}^{2}, \ldots, \quad w^{(2 m-3)}\left(z_{0}\right)=(v / u)^{(2 m-3)}\left(z_{0}\right)$, where we assume $u^{\prime \prime}\left(z_{0}\right)=u_{0}^{\prime^{2}} / u_{0}$. We shall construct a solution of system (6) applying the formula

$$
u_{1}(z)=u_{0} \exp \left(\left(z-z_{0}\right) \frac{u_{0}^{\prime}}{u_{0}}-\int_{z_{0}}^{z} d z \int_{z_{0}}^{z} w^{2}(z) d z\right), v_{1}(z)=u_{1}(z) w(z) .
$$

It is not difficult to see that the solution $\left(v_{1}(z), u_{1}(z)\right)$ satisfies to the same initial conditions, as $(v(z), u(z))$. In view of this by virtue of uniqueness 
$\left(v_{1}(z), u_{1}(z)\right) \equiv(v(z), u(z))$ and from the second parity of (11) statement of the theorem follows.

THEOREM 3. Any meromorphic solution $w(z)$ of equation $\left({ }_{m} P_{2}\right)$ is represented in the form $w(z)=v(z) / u(z)$, where $(v(z), u(z))$ is the corresponding entire solution of system (6), determined up to the factor $\exp (a z+b)$.

$\mathrm{P}$ r o o f. If $w(z) \equiv 0$ at $\delta=0$, we shall take the entire solution of system (6) $(v(z), u(z))=(0, \exp (a z+b))$. Let $w(z) \not \equiv 0$. Then there exists such $z_{0}$, that $w\left(z_{0}\right) \neq 0, w\left(z_{0}\right) \neq \infty$. Let us put

$$
u(z)=\exp \left(-\int_{z_{0}}^{z} d z \int_{z_{0}}^{z} w^{2} d z\right), \quad v(z)=w(z) u(z) .
$$

These functions are entire solutions of system (6), and $w(z)=v(z) / u(z)$. But by virtue of $(9) w(z)$ is also expressed through the solution

$$
(\tilde{v}(z), \tilde{u}(z))=(v(z) \exp (a z+b), u(z) \exp (a z+b))
$$

The theorems 2 and 3 establish one-to-one correspondence between the meromorphic solutions of equation $\left({ }_{m} P_{2}\right)$ and the entire solutions of system (6).

\section{REFERENCES}

[1] V. I. Gromak. On general representation of solutions of the Painleve equation. Differential Equations, 3(5), 1997, P. 614 - 617. (in Russian).

[2] P. Painleve. Mémoire sur les équation différentielles dont l'intégrale générale est uniforme. Bull. Soc. Math. France, 28, 1900, P. $201-261$.

[3] V. V. Golubev. To the theory of Painleve Equations. Math. Sb., 28(2), 1912, P. 323 - 349. (in Russian).

[4] V. I. Gromak. To the theory of higher anologeus of Korteveg - de Vries equations. Dep. in "Differential Equations", No 3641 - 86. (in Russian). 